\title{
Colonización por Pneumocystis jirovecii en la enfermedad pulmonar obstructiva crónica
}

\section{Colonization by Pneumocystis jirovecii in Chronic Obstructive Pulmonary Disease}

\author{
Alejandra Cañas A. ${ }^{a}$ \\ Pontificia Universidad Javeriana, Colombia \\ JaVier R. Garzón H. ${ }^{b}$ \\ Pontificia Universidad Javeriana, Colombia \\ Catalina Hernández F.c \\ Pontificia Universidad Javeriana, Colombia \\ Juan F. Burbano G. ${ }^{\mathrm{d}}$ \\ Pontificia Universidad Javeriana, Colombia \\ José E. Cita P. \\ Pontificia Universidad Javeriana, Colombia \\ Claudia M. Parra G. ${ }^{\mathrm{f}}$ \\ Pontificia Universidad Javeriana, Colombia
}

a Neumología, Hospital Universitario San Ignacio. Directora del Departamento de Medicina Interna de la Pontificia Universidad Javeriana, Bogotá, Colombia.

b Infectología, Hospital Universitario San Ignacio. Profesor de la Facultad de Medicina de la Pontificia Universidad Javeriana, Bogotá, Colombia.

c Medicina Interna, Hospital Universitario San Ignacio. Profesora de la Facultad de Medicina de la Pontificia Universidad Javeriana, Bogotá, Colombia.

d Medicina Interna. Hospital Universitario San Ignacio, Pontificia Universidad Javeriana, Bogotá, Colombia.

e Medicina Interna, Hospital Universitario San Ignacio-Pontificia Universidad Javeriana, Bogotá, Colombia.

f Micología, Departamento de Microbiología, Pontificia Universidad Javeriana, Bogotá, Colombia.

\section{Declaración de conflictos de interés: ninguno}

Fuente de financiamiento: Proyecto Id 00006339 , financiado por la Vicerrectora de Investigación de la Pontificia Universidad Javeriana y la Oficina de Investigación del Hospital Universitario San Ignacio.

Cómo citar: Cañas A, Garzón JR, Hernández C, Burbano JF, Cita JE, Parra-Giraldo CM. Colonización por Pneumocystis jirovecii en la enfermedad pulmonar obstructiva crónica. Univ. Med. 2018; 59(3). doi: https://doi.org/10.11144/Javeriana.umed 59-3.pneu

\section{RESUMEN}

La enfermedad pulmonar obstructiva crónica (EPOC) es una enfermedad prevalente en la población colombiana, que característicamente tiene un deterioro progresivo y altera la calidad de vida de los pacientes. Solo del 15\% al 20\% de los pacientes con antecedente de tabaquismo desarrollan la enfermedad, por lo que factores ambientales y genéticos adicionales influyen en su progresión. De las causas infecciosas que han tomado importancia, el Pneumocystis jirovecii, un hongo ubicuo que entra en contacto con la vía aérea de los humanos desde la infancia, es causa de neumonía en pacientes inmunosuprimidos. Se han descrito tasas elevadas de colonización en pacientes con EPOC, que aumentan con la severidad de la enfermedad. EPOC e infección por $P$. jirovecii parecen compartir una respuesta inmunológica similar, lo cual podría explicar el papel de la colonización por el hongo en su progresión y gravedad.

Palabras clave

Pneumocystis jirovecii ; enfermedad pulmonar obstructiva crónica; inmunología.

\begin{abstract}
Chronic obstructive pulmonary disease (COPD) is a prevalent disease in our population, which characteristically has a progressive deterioration and alters the quality of life of patients. Only $15-20 \%$ of patients with a history of smoking develop the disease, so there are additional environmental and genetic factors that influence the progression of the disease. Of the infectious causes that have taken importance is Pneumocystis jirovecii, this is a ubiquitous fungus that comes into contact with the airway of humans since childhood and is a cause of pneumonia in immunosuppressed patients. In addition, high rates of colonization have been reported in patients with COPD, which increase with the severity
\end{abstract}


of the disease. COPD and P. jirovecii infection appear to share a similar immune response, which may explain the role of fungal colonization in the progression and severity of the disease.

Keywords

Pneumocystis jirovecii ; pulmonary disease chronic obstructive; immunology.

\section{Introducción}

La enfermedad pulmonar obstructiva crónica (EPOC) tiene una prevalencia en la población latinoamericana del 14\% (1), y del 8,9\% en Colombia (2). En el 2015 se reportó como la cuarta causa de muerte por enfermedades no transmisibles en Colombia (3) y se estima que en el año 2020 será la quinta causa de discapacidad en el mundo por años de vida ajustados por discapacidad (4). A la EPOC se han asociado factores como el humo del cigarrillo y la exposición laboral a partículas tóxicas —específicamente en nuestro medio a la biomasa-, lo cual desencadena una respuesta inflamatoria crónica de las vías respiratorias y destrucción del parénquima pulmonar, generando obstrucción fija de la vía aérea y disminución de la retracción elástica pulmonar (4) . Los pacientes presentan disnea y tos con expectoración crónica; cursan, además, con deterioro de la capacidad funcional, menor calidad de vida, exacerbaciones frecuentes y mayor riesgo de muerte. Por cada exacerbación que se acompaña de acidosis respiratoria, se estima una mortalidad intrahospitalaria del $10 \%$, y en aquellos que requieren ventilación mecánica, alcanzan una mortalidad al año hasta del $40 \%$, lo que convierte a la EPOC en un problema de salud pública (5).

Se han venido estudiando diferentes factores asociados a estas exacerbaciones y en los últimos años estudios en animales y humanos han documentado la colonización por Pneumocystis jirovecii como generador de cambios en la arquitectura pulmonar que llevan a obstrucción bronquial (6).

En este artículo, revisaremos la biología y la respuesta inmunológica producida por la infección por P. jirovecii y cómo estas características podrían explicar el efecto que la colonización por el hongo tiene en los pacientes con EPOC no inmunosuprimidos como uno de los factores perpetuadores de respuesta inflamatoria pulmonar que repercute en la progresión de la enfermedad.

\section{Biología del Pneumocystis jirovecii}

El hongo P. jirovecii fue descrito por Carlos Chagas, en 1909, como parte del ciclo vital del Trypanosoma cruzi (7), denominado inicialmente como Schizotrypanum cruzi (8). Después el matrimonio Delanöe lo nombró Pneumocystis carinii, en 1912: pneumo, por su tropismo o afinidad por el pulmón; cystis, por su morfología quística, y carinii, en honor a Antonio Carini, quien ya lo había documentado en pulmones de rata en $1910(7,8)$. A pesar de que se han encontrado ácidos nucleicos en el aire en cultivos de manzanas y en el agua estancada, se desconoce un nicho diferente a los mamíferos (7). Solo hasta 1976 se planteó la hipótesis de que la especie de Pneumocystis, que afectaba al hombre, era diferente de la que infectaba a las ratas, a pesar de no poseer diferencias morfológicas distinguibles. El nuevo nombre propuesto fue Pneumocystis jirovecii en honor al científico checo Otto Jirovec, quien en 1951 relacionó el patógeno con la neumonía que aparecía en niños prematuros y malnutridos (9).

En 1988, con estudios moleculares se determinó el Pneumocystis como miembro del reino fungi. $\mathrm{Su}$ ubicación taxonómica actual es en el reino: Fungi; filo: Ascomycota; clase: pneumocystidomycetes; orden: Pneumocystidales, y familia: Pneumocystidacae. Hoy, conocemos a Pneumocytis jiroveccii como un hongo atípico, extracelular, ubicuo, unicelular, no cultivable y con estenoxenismo, es decir, con especificidad entre el hospedero y el microorganismo (10).

Pese a estos avances, el conocimiento de la biología y ciclo de vida del Pneumocystis ha sido limitado, debido a la ausencia de un método in vitro de cultivo (11), lo que ha dificultado definir el ciclo de vida completo. Los datos conocidos provienen del establecimiento de varios modelos animales en diferentes mamíferos (12). Por otro 
lado, se han identificado dos formas de ciclo celular: la primera es el de trofozoito o forma trófica (de 1 a 4 micrómetros), que se puede identificar con la tinción de Giemsa, donde el núcleo se visualiza rojo, y el citoplasma, azul. La segunda forma es el quiste de 5 a 8 micrómetros que se identifica mediante tinciones de plata metenamina (13). Durante la infección pulmonar, las formas tróficas predominan sobre las quísticas en una relación de 10:1; entre estas dos formas de ciclo celular se encuentran estadios intermedios de la forma quística (temprana, intermedia y prequiste tardío), difíciles de ver mediante microscopia de luz y que se han identificado mediante microscopia electrónica (7).

La pared celular del hongo es rica en el polisacárido $\beta-1,3$ glucano y colesterol; además de contener residuos de glucosa/ manosa, $\mathrm{N}$-acetilglucosamina y galactosa/ $\mathrm{N}$ acetilgalactosamina. A diferencia del resto de hongos, carece de ergosterol, razón por la cual presenta resistencia innata a los antifúngicos convencionales como anfotericina B y a los azoles. Sus proteínas tienen características inmunogénicas diferentes. Dentro de las más estudiadas está la glucoproteína principal de superficie o gpA, con capacidad para interactuar con proteínas del hospedero como la fibronectina, la vitronectina y la laminina; así mismo, está el surfactante que, junto al receptor de manosa, facilita la relación con las células del hospedero y su actividad inmunogénica (14). Otro de los complejos antigénicos identificado en la superficie de Pneumocystis derivado de la rata es la familia de genes PRT1, también conocida como la familia de multigenes KEX, que codifica las proteínas que participan en la variación antigénica del microorganismo (15). De su contenido genético, se ha estimado que, por lo menos, el 10\% de su material génico es no codificable, lo cual podría sustentar, en parte, su dependencia del hospedero para realizar muchas funciones vitales (16).

La ruta de infección es la respiratoria, con transmisión por aerosoles de hospedero a hospedero (17). Se sospecha que la colonización ocurre desde edades tempranas, ya que se han encontrado anticuerpos circulantes desde los 7 meses de vida (6). Lo más aceptado en la actualidad es que la infección se presenta por exposiciones de novo, más que por reactivaciones de infecciones previas. Esto obedece a que se han documentado pacientes con VIH y neumonía por P. jirovecii con episodios repetidos de infección por genotipos diferentes (18). Adicionalmente, se han detectado microorganismos con mutaciones en el gen de la dihidrofolatoreductasa, aislados de pacientes sin exposición previa a trimetroprima/ sulfametoxazol, lo cual también sugiere la adquisición de novo por transmisión de humano a humano (18). Usualmente, el hongo se encuentra restringido a los alvéolos pulmonares, aunque en alguna ocasión se han encontrado en otros tejidos en pacientes con sida (19).

\section{Respuesta inmunológica}

Los macrófagos alveolares son la primera línea de defensa contra el $P$. jirovecii, con la ventaja de eliminar tanto el trofozoito como el quiste, al fagocitarlo y degradarlo por fusión fagolisosomal. Estos reconocen patrones moleculares asociados a patógenos a través de receptores de reconocimiento de patrones (RRP). El primero de ellos es el receptor de manosa que, de forma similar a otros hongos, se une a la manosa de la pared del Pneumocystis (glicoproteína principal de superficie). El segundo RRP del macrófago que se visualiza en la figura 1 es la dectina-1, la cual reconoce al $\beta$-glucano de la pared del hongo y genera la endocitosis y fagocitosis, además de la activación del factor de transcripción nuclear potenciador kappabeta (NF-kb), que resulta en la producción de citocinas inflamatorias, interleucina 8 (IL-8), metaloproteinasas y proteína inflamatoria de los macrófagos (PIM-2) (figura 1). 


\section{Figura 1}

Los quistes del P. jirovecii entran en contacto con el neumocito tipo I, fibronectina y surfactante, donde las PS A y D se unen al Pneumocystis facilitando su identificación por los macrófagos; éstos reconocen al hongo por medio de RRP tipo TLR 2 y 4, Rec de Manosa y Dectina-1; con lo cual se liberan interleucinas, TNF- $\alpha$, PIM-2 y MMP-12 vía NF$x B$, contribuyendo a la destrucción del hongo, pero también al daño pulmonar. En la fagocitosis el hongo es destruido por la producción de $\mathrm{ON}, \mathrm{H}_{2} \mathrm{O}_{2}$ y RNIs, respuesta condicionada por INF- $\gamma$ y TNF$\alpha$. Las células dendríticas reconocen los antígenos del hongo y migran a los ganglios linfáticos donde los presentan para activar la respuesta celular y humoral. Los LT CD4 generan respuesta de memoria y coordinan la respuesta inflamatoria, además reclutan y activan otras células efectoras, la IL-12 (producida por las células dendríticas) promueve la respuesta hacia Th1, con producción de INF- $\gamma$, el cual es responsable de la actividad citotóxica de los $\mathrm{LT} \mathrm{CD}{ }^{+}$, potenciación de los mecanismos efectores de las células presentadoras de antígenos y diferenciación de los LB a células plasmáticas para la producción de anticuerpos contra gpA y KEX1. Los LT CD8 ${ }^{+}$activados, al igual que la liberación masiva de citocinas genera quimiotaxis de neutrófilos, los cuales no destruyen al hongo, pero sí afectan el parénquima pulmonar; hay proliferación de neumocitos tipo I y exudado alveolar espumoso rico en eosinófilos, llevando a la producción de moco y la destrucción de las paredes alveolares conduciendo a bronquitis y enfisema respectivamente.

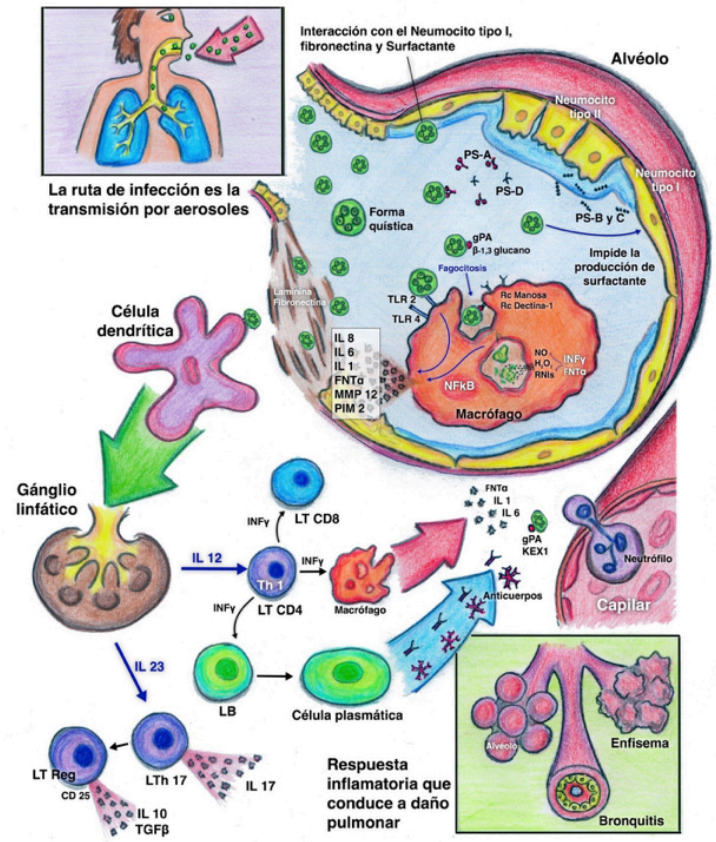

PS: proteínas del surfactante; RRP: receptores de reconocimiento de patrones; TLR: Toll-like Receptors; Rec: receptores; gpA: glicoproteína principal de superficie; NF- $\kappa$ B: factor de transcripción nuclear kappa-beta; IL: interleucinas; TNF- $\alpha$ : factor de necrosis tumoral alfa; PIM-2: proteína inflamatoria de los macrófagos; MMP-12: metaloelastasa de macrófagos; $\mathrm{ON}$ : óxido nítrico; $\mathrm{H} 2 \mathrm{O} 2$ : peróxido de hidrógeno; RNI: intermediarios reactivos de nitrógeno; INF $\gamma$ : interferón gamma; LT: linfocitos T; LB: linfocitos B; KEX1: kexina 1; TGF $\beta$ : factor de crecimiento transformante beta.

Otros RRP reconocidos que participan en la respuesta inflamatoria, mas no en la fagocitosis, son los receptores TLR (de su siglas en inglés TollLike Receptors), entre los que se destacan TLR-4 y principalmente TLR-2, que influye también en la producción de citosinas, factor de necrosis tumoral alfa (TNF- $\alpha$ ), IL-8 y proteína inhibidora de macrófagos 2 (PIM-2) (20).

Posterior a la fagocitosis, por medio de radicales libres y productos reactivos del oxígeno, principalmente peróxido de hidrogeno, se destruye el hongo. Así mismo, participan el óxido nítrico e intermediarios reactivos de nitrógeno, respuesta condicionada por la presencia de interferón gamma (INF- $\gamma$ ) y TNF- $\alpha$ (20).

$P$. jirovecii cuenta con diversos mecanismos para evadir el sistema inmune, entre estos se destacan la variabilidad antigénica de la gpA 
y la inducción de apoptosis de los macrófagos alveolares por medio de la producción de poliaminas intracelulares y de caspasa-9 (20).

Otros factores adicionales que participan en la respuesta contra el Pneumocystis son las proteínas del surfactante (PS), de las cuales existen dos grupos: las hidrofóbicas (PS-B y PS-C), que participan en la generación de tensión superficial en el alvéolo, y las hidrofílicas (PS-A y PS$D)$, que participan en la respuesta inmunitaria innata. El P. jirovecii disminuye las PS-B y PS$\mathrm{C}$, lo que probablemente contribuye a la falla respiratoria hipoxémica (figura 1). Las proteínas PS-A y PS-D se unen a las moléculas de manosa del Pneumocystis y con ello facilitan el reconocimiento por los macrófagos alveolares (20).

Como se ve en la figura 1, otras células participantes en la respuesta inmune innata son las dendríticas, que se encuentran en el tejido intersticial y peribronquial y cumplen un papel importante en la presentación de antígenos, al migrar hacia los ganglios linfáticos donde activan la respuesta de linfocitos $\mathrm{T}$ a la infección. Los neutrófilos, a diferencia de su trabajo en la infección por otros microorganismos, no participan en la defensa contra el hongo, pero sí se correlacionan y son partícipes en la inflamación, destrucción del parénquima pulmonar y severidad de la enfermedad (20).

Dentro de las células clave en la respuesta adaptativa contra Pneumocystis se encuentran los linfocitos T-CD4, que generan la respuesta de memoria y coordinan la respuesta inflamatoria. Además, reclutan y activan otras células efectoras, que son responsables de la eliminación final del microorganismo. Una subpoblación de linfocitos T-CD4 positivos para CD25 son claves para regular la respuesta inflamatoria al hongo, pues se ha encontrado una correlación entre la depleción de estas células $\mathrm{T}$ y la severidad de la enfermedad (20).

Los linfocitos T-CD8 tienen un papel dicotómico dependiendo de la respuesta generada ante la presencia del hongo. Bajo el estímulo de INF- $\gamma$ cumplen un papel citotóxico, ya que eliminan el patógeno y protegen al hospedero en estados de depleción de células
CD4. Pero si su función no es citotóxica, su presencia produce daño pulmonar acelerado y deterioro de la función pulmonar, a través de receptores del TNF- $\alpha$ en el tejido pulmonar, lo que sube las concentraciones de citocinas que producen quimiotaxis de neutrófilos y monocitos (20).

En cuanto a la inmunidad humoral, se ha documentado que los anticuerpos específicos contra $P$. jirovecii tienen un papel importante en la defensa contra el hongo. Está descrito que ratones con defectos en los linfocitos $\mathrm{B}$ tienen una respuesta exagerada contra la infección por el microorganismo; en humanos se ha documentado aumento de los episodios de neumonía por el hongo, al recibir medicamentos que inhiben los linfocitos B. Adicionalmente, la evidencia de respuesta serológica desde edades tempranas ha reafirmado el rol fundamental de la inmunidad humoral en la defensa contra el organismo. El anticuerpo predominante es de tipo IgG, pero también se han documentado anticuerpos de tipo IgM. Los estudios serológicos se han dirigido principalmente a dos antígenos: la glicoproteína principal de superficie, en la cual se han descrito arreglos genómicos con variabilidad antigénica consecuente, lo que otorga al hongo un mecanismo de defensa para evadir el sistema inmunológico como se había planteado previamente. El segundo antígeno es una proteasa llamada Kexina (Kexina-1), que participa en la proteólisis de los antígenos de superficie del Pneumocystis. Estudios han demostrado que los anticuerpos de tipo IgG a este antígeno generan fagocitosis mediada por la porción $F_{c}$ y, además, activación del complemento $(6,20)$.

El INF-g producido por los linfocitos $\mathrm{T}$ CD4 es importante en la defensa contra microorganismos oportunistas como P. jirovecii, ya que controla la respuesta inflamatoria generada por la infección; por ende, involucrada en la severidad y las secuelas del parénquima pulmonar. Adicionalmente, el TNF- $\alpha$ secretado por los macrófagos alveolares ejerce un papel protector contra el Pneumocystis, ya que esta citosina, liberada en conjunto con IL-1 e IL-6, induce la producción de otras citocinas e INF- $\gamma$ 
que promueven aún más la respuesta inflamatoria contra el hongo. También participa de esta respuesta la IL-12, producida principalmente por los macrófagos y las células dendríticas, que promueve la respuesta celular hacia linfocitos Th-1, y por ende, para la producción de INF-g (21).

Un componente fundamental de la respuesta inflamatoria al hongo es el eje IL-23/IL-17 (figura 1). La IL-17 es producida por las células Th17, y la IL-23, por las células presentadoras de antígenos, que estimulan la producción de IL-17. Por tanto, la falta de estas interleucinas induce la eliminación del hongo. Toda esta respuesta inflamatoria dirige la producción de exudado alveolar rico en eosinófilos, con presencia de membranas hialinas, edema y fibrosis intersticial. Por su parte, el hospedero responde con proliferación de los neumocitos tipo II e infiltrado de células mononucleares (22).

\section{Colonización}

Se define colonización como la detección del $P$. jirovecii en pacientes asintomáticos o sin signos sugestivos de neumonía. Esta detección se realiza por métodos moleculares, ya que los tradicionales por visión directa detectan solo altas cargas del microorganismo $(23,24)$.

Las seroprevalencias de colonización por Pneumocystis aumentan con la edad: un 52\% a los 6 años y hasta un 80\% a los 13 años (25). En especímenes obtenidos por autopsias de niños que murieron en casa, se ha detectado el hongo hasta en un 33\% por métodos de tinción directa y hasta en un $52 \%$ por métodos moleculares $(24,26,27)$.

Un estudio realizado en 50 adultos inmunocompetentes sin enfermedad pulmonar analizó la presencia de $P$. jirovecii en muestras de lavado orofaríngeo por medio de la reacción en cadena de la polimerasa (PCR) anidada. Encontró que el 20\% de los sujetos estaban colonizados por el hongo. A 9 de los 12 pacientes colonizados se les pudo hacer seguimiento a los 6 meses y se halló que sólo en 2 persistía la colonización (28). En otro estudio llevado a cabo en Chile, de individuos llevados a autopsia médico-legal, se documentó la presencia del hongo por medio de PCR en el 65\%, sin que este hallazgo tuviera relación con la causa de muerte (29).

La prevalencia de colonización en pacientes con infección por $\mathrm{VIH}$ es del $44 \%$ al $69 \%$ $(30,31,32)$. La relación de la colonización con el número de linfocitos T-CD4 es debatida, pues su correlación no se reproduce en los diferentes estudios, dado que la colonización también ocurre en aquellos pacientes con altos niveles de $\mathrm{CD} 4$, en quienes reciben terapia antirretroviral e incluso en pacientes con profilaxis contra Pneumocystis. Por otra parte, el ser fumador y vivir en la ciudad se han encontrado como factores de riesgo para colonización (32).

\section{Colonización y enfermedad pulmonar crónica}

La presencia de enfermedades pulmonares crónicas también se ha asociado con la predisposición para estar colonizado (24). Probst y cols. (33) evaluaron la detección de $P$. jirovecii por métodos moleculares en pacientes con diferentes enfermedades pulmonares y encontraron que más del $21 \%$ estaban colonizados. De estos, el 40\% tenía EPOC; el 20\%, cáncer pulmonar, y el $7 \%$, fibrosis quística (33). En un estudio en pacientes con fibrosis quística se detectó, por medio de PCR, una colonización hasta del 20\% $(34,35,36)$. En pacientes con enfermedad pulmonar intersticial difusa se encontró colonización hasta del 34\%, especialmente en pacientes fumadores y aquellos que recibían corticosteroides $(37,38)$. Diferentes series han informado sobre la prevalencia de colonización por el hongo en pacientes con EPOC entre el $13 \%$ y el $55 \%$ por PCR anidada en esputo inducido $(37,39,40)$.

En un reciente estudio publicado por Wang y cols. (38), donde evaluaron a 98 pacientes con enfermedad pulmonar, de los cuales 47 tenían EPOC y 25 enfermedad pulmonar intersticial difusa, se encontró una colonización de un 53,2\%, evaluado por una técnica de 
amplificación isotérmica mediada por asas para identificar un gen diana del Pneumocystis (41).

El efecto de la colonización se postula en varios escenarios. Primero, desconocemos si la neumonía por $P$. jirovecii está precedida de periodos de colonización, y si se puede modificar con profilaxis. Segundo, si los individuos colonizados sirven de reservorio para la transmisión del microorganismo. Y, por último, la sola presencia del hongo genera una respuesta inflamatoria local y sistémica que puede influir en el deterioro de la función pulmonar de estos pacientes (42) y, por tanto, que el tratamiento pueda evitar la progresión del daño pulmonar. Sin embargo, un estudio en animales infectados por retrovirus, donde se redujo la prevalencia de colonización con trimetoprima/sulfametoxazol no hubo mejoría de la función pulmonar (43). No obstante, faltan estudios en humanos, sin inmunosupresión y enfermedades pulmonares para poder generalizar esta conclusión en esta subpoblación.

Pneumocystis jirovecii y enfermedad pulmonar obstructiva crónica

Es bien conocido el papel del tabaquismo o la exposición a humo de leña en la fisiopatología de la EPOC; sin embargo, solo del 15\% al 20\% de los fumadores desarrollan la enfermedad, por lo cual se postulan otros factores que influyen en el curso natural de la enfermedad, tanto de tipo genético como ambiental. En estos últimos, los microorganismos se han propuesto como generadores de un proceso inflamatorio persistente que influye en el deterioro acelerado de la función pulmonar (5).

Como se mencionó, se ha detectado mayor prevalencia de colonización de $P$. jirovecii en pacientes con enfermedades pulmonares, sobre todo en pacientes con EPOC. Se afirma que la elevada detección del hongo en pacientes sin neumonía sea un hallazgo asociado a la enfermedad estructural, inmunosupresión por corticosteroides o exposición a humo de cigarrillo; sin embargo, en los últimos años, estudios en animales y humanos han documentado un papel importante de la colonización por $P$. jirovecii, al asociar la respuesta inflamatoria local y sistémica con cambios estructurales y deterioro en la función pulmonar, similar a la presentada por los pacientes con EPOC (22).

En primera instancia, se ha determinado que los pacientes con infección por VIH colonizados por $P$. jirovecii tienen mayor riesgo de obstrucción de la vía aérea (OR: 8,8), a diferencia de los pacientes no colonizados, incluso después de ajustar por la historia de tabaquismo de cada paciente (44).

En otro estudio realizado por Morris y cols. (45) en pacientes con EPOC sin VIH se documentó la asociación de colonización por $P$. jirovecii con la severidad de la enfermedad, independientemente del antecedente de tabaquismo y de inmunosupresión. Puntualmente, en este estudio se encontró que el $37 \%$ de los pacientes con EPOC severo y que el 5\% con enfermedad leve estaban colonizados, a diferencia del 9\% de pacientes con otras enfermedades pulmonares (45), lo que confirmó no solo la mayor prevalencia de colonización en pacientes con EPOC, sino también la asociación de dicha colonización con la severidad de la enfermedad.

¿Cómo la colonización por P. jirovecii influiría en la historia natural de los pacientes con enfermedad pulmonar obstructiva crónica?

La principal alteración que se encuentra en la vía aérea de pequeño calibre de pacientes con EPOC consiste en la presencia de infiltrado inflamatorio y remodelación bronquial, que aumenta el grosor de la pared bronquial, reduciendo el diámetro y aumentando la resistencia al flujo de aire (figura 1). El linfocito T-CD8 es la principal célula presente en la vía aérea y el parénquima pulmonar de pacientes con EPOC, motivo por el cual la cantidad de estas células se correlaciona con el grado de obstrucción al flujo de aire. Los linfocitos T-CD4 están aumentados en los pacientes con EPOC, y la cantidad de estos que secretan INF-g se correlaciona con el grado de 
obstrucción de la vía aérea; esto sugiere que tanto las células T-CD4 como las CD8 desempeñan un papel importante en la fisiopatología de la EPOC, siendo la expresión del fenotipo Th1 la clave que promueve la migración transendotelial de células inflamatorias al sitio implicado. En pacientes con EPOC severo se ha documentado la persistencia de linfocitos T-CD4 Th1 activados, oligoclonales, linfocitos B y linfocitos T-CD8 que pueden persistir por años incluso después de haber dejado de fumar (46).

Al mirar con detalle el impacto de $P$. jirovecii en la historia natural de la enfermedad, estudios en animales han determinado que el microorganismo genera una respuesta inflamatoria similar a la encontrada en pacientes con EPOC. En un estudio realizado en un modelo de sida en primates se documentó que la colonización por $P$. jirovecii no solo estaba asociada con el deterioro de la función pulmonar, sino de manera paralela con el aumento de la respuesta inflamatoria tipo Th2, al hallarse un aumento en las concentraciones de interleucinas 4, 5, 6 y factor estimulador de colonias granulocíticas en los lavados broncoalveolares de los primates colonizados versus los no colonizados, así como un aumento en el tejido linfoide asociado a bronquios (47); sin embargo, no se evidenció progresión del deterioro pulmonar, lo que sugiere la necesidad de una noxa adicional para disminuir la función pulmonar en humanos (48).

Por otro lado, en estudios realizados en humanos con VIH se ha documentado un aumento en los niveles de metaloelastasa de macrófagos en esputo de los pacientes colonizados, independientemente de la exposición a tabaco, lo que sugiere una relación entre la colonización y la EPOC, pues es bien sabido el papel que cumple la metaloelastasa de macrófagos en la fisiopatología de la enfermedad, al documentarse una sobreexpresión y un desequilibrio entre proteasas y antiproteasas principalmente en pacientes fumadores con EPOC (44).

Adicionalmente, se ha descrito la activación de vías moleculares y genes reguladores relacionados con el proceso inflamatorio estudiado en pacientes con EPOC y se ha estudiado que aquellos pacientes con colonización por $P$. jirovecii tienen activación de ligandos como CXCL 9-11 y CXCR3 ante la presencia de INF-a in vitro (al activarse la vía de Th1) y, posteriormente, ante la presencia de las citocinas proinflamatorias descritas previamente. De este modo, la colonización por Pneumocystis es un posible activador de la respuesta inflamatoria pulmonar, que induce a los macrófagos a producir metaloproteinasa, clave en mecanismos fisiopatológicos como la formación de enfisema pulmonar (22).

Además de la respuesta inflamatoria local pulmonar, se ha demostrado que aumenta la respuesta inflamatoria sistémica dada por niveles elevados de IL-6, IL-8 y TNF- $\alpha$ en pacientes con EPOC colonizados por P. jirovecii $(40,49)$.

Como ya se discutió, el control inmunológico de la infección por P. jirovecii depende tanto de la actividad de los linfocitos T-CD4 como de la producción de anticuerpos. Los anticuerpos dirigidos contra la proteasa Kexina parecen ser particularmente importantes en el control de la infección en modelos murinos. En primates no humanos se ha demostrado que estos anticuerpos predicen la susceptibilidad a la colonización tras inducción de estados de inmunosupresión (50).

La relación entre los títulos de anticuerpos anti-KEX1 y el grado de EPOC también fue examinada en un estudio de fumadores VIH negativos. El $60 \%$ de los participantes tenía anticuerpos anti-KEX1, y se determinó que los títulos bajos eran predictores de mayor severidad de la obstrucción de la vía aérea (51). Estos hallazgos no solo relacionan un mayor riesgo de colonización cuando hay alteraciones de la respuesta humoral, sino que también sugieren la relación de colonización por $P$. jirovecii con deterioro de la función pulmonar y severidad de la EPOC (figura 1).

\section{Conclusión}

En años recientes se han aclarado algunos aspectos de la interrelación entre los humanos y el Pneumocystis jirovecii. Actualmente, se 
ha reconocido como causa importante de neumonía en pacientes con inmunosupesión celular y humoral; sin embargo, se ha demostrado colonización en pacientes inmunocompetentes con diversos tipos de enfermedad estructural pulmonar, principalmente en EPOC, en quienes se han hallado tasas aún mayores de colonización que en aquellos quienes cursan con mayor compromiso de la función pulmonar.

Además, esta colonización parece ser importante en el incremento de la respuesta inflamatoria local y sistémica en pacientes colonizados, que podría contribuir al daño progresivo del parénquima pulmonar. No obstante, aún falta mucha información que nos permita esclarecer qué labor cumple P. jirovecii en la fisiopatología e historia natural de la EPOC. Podría corresponder a un epifenómeno, pero vemos cómo los datos presentados de diferentes autores apuntan a la posibilidad de que este microorganismo pueda ser uno de los factores perpetuadores de la respuesta inflamatoria en el árbol bronquial, que desde el punto de vista clínico se traduciría en progresión de la enfermedad y mayor tasa de exacerbaciones.

\section{Referencias}

1. Menezes AM, Pérez-Padilla R, Jardim JR, Muino A, López MV, Valdivia G, et al. Chronic obstructive pulmonary disease in five Latin American cities (the PLATINO study): Aa prevalence study. Lancet (London, England). 2005;366(9500):1875-81.

2. Caballero A, Torres-Duque CA, Jaramillo C, Bolívar F, Sanabria $\mathrm{F}$, Osorio $\mathrm{P}$, et al. Prevalence of COPD in five Colombian cities situated at low, medium, and high altitude (PREPOCOL study). Chest. 2008;133(2):343-9.

3. Collaborators GBDDaH. Global, regional, and national disabilityadjusted life-years (DALYs) for 315 diseases and injuries and healthy life expectancy (HALE), 1990-2015: a systematic analysis for the Global Burden of Disease Study 2015. Lancet (London, England). 2016;388(10053):1603-58.

4. Vestbo J, Hurd SS, Agusti AG, Jones PW, Vogelmeier C, Anzueto A, et al. Global strategy for the diagnosis, management, and prevention of chronic obstructive pulmonary disease: GOLD executive summary. Am J Respir Crit Care Med. 2013;187(4):347-65.

5. Vestbo J, Hurd SS, RodriguezRoisin R. The 2011 revision of the global strategy for the diagnosis, management and prevention of COPD (GOLD)--why and what? Clin Respir J. 2012;6(4):208-14.

6. Morris A, Norris KA. Colonization by Pneumocystis jirovecii and its role in disease. Clin Microbiol Rev. 2012;25(2):297-317.

7. Thomas CF, Jr., Limper AH. Current insights into the biology and pathogenesis of Pneumocystis pneumonia. Nat Rev Microbiol. 2007;5(4):298-308.

8. Calderón Sandubete E, de Armas Rodríguez Y, Capo de Paz V. Pneumocystis jirovecii: one hundred years of history. Rev Cubana Med Trop. 2011;63(2):97-116.

9. Frenkel JK. Pneumocystis jiroveci n. sp. from man: Morphology, physiology, and immunology in relation to pathology. National Cancer Institute Monograph. 1976;43:13-30.

10. Mazars E, Dei-Cas E. Epidemiological and taxonomic impact of Pneumocystis biodiversity. FEMS immunology and medical microbiology. 1998;22(1-2):75-80.

11. Sloand E, Laughon B, Armstrong M, Bartlett MS, Blumenfeld W, Cushion $\mathrm{M}$, et al. The challenge of 
Pneumocystis carinii culture. J Eukariot Microbiol. 1993;40(2):188-95.

12. Aliouat-Denis CM, Martínez A, Aliouat el M, Pottier M, Gantois N, Dei-Cas E. The Pneumocystis life cycle. Memorias do Instituto Oswaldo Cruz. 2009;104(3):419-26.

13. Burgess JW, Kottom TJ, Limper AH. Pneumocystis carinii exhibits a conserved meiotic control pathway. Infect Immun. 2008;76(1):417-25.

14. Kottom TJ, Kennedy CC, Limper AH. Pneumocystis PCINT1, a molecule with integrin-like features that mediates organism adhesion to fibronectin. Mol Microbiol. 2008;67(4):747-61.

15. Ambrose HE, Keely SP, Aliouat EM, Dei-Cas E, Wakefield AE, Miller RF, et al. Expression and complexity of the PRT1 multigene family of Pneumocystis carinii. Microbiology (Reading, England). 2004;150(Pt 2):293-300.

16. Stringer JR, Cushion MT. The genome of Pneumocystis carinii. FEMS Immunology and Medical Microbiology. 1998;22(1-2):15-26.

17. Dumoulin A, Mazars E, Seguy N, Gargallo-Viola D, Vargas S, Cailliez JC, et al. Transmission of Pneumocystis carinii disease from immunocompetent contacts of infected hosts to susceptible hosts. Eur J Clin Microbil Infet Dis. 2000;19(9):671-8.

18. Hauser PM, Nahimana A, Taffe P, Weber R, Francioli P, Bille $\mathrm{J}$, et al. Interhuman transmission as a potential key parameter for geographical variation in the prevalence of Pneumocystis jirovecii dihydropteroate synthase mutations. Clin infect Dis. 2010;51(4):e28-33.

19. Kovacs JA, Masur H. Evolving health effects of Pneumocystis: one hundred years of progress in diagnosis and treatment. JAMA. 2009;301(24):2578-85.

20. Kelly MN, Shellito JE. Current understanding of Pneumocystis immunology. Future Microbiol. 2010;5(1):43-65.

21. Iriart $X$, Witkowski $B$, Courtais C, Abbes S, Tkaczuk J, Courtade M, et al. Cellular and cytokine changes in the alveolar environment among immunocompromised patients during Pneumocystis jirovecii infection. Med Mycol. 2010;48(8):1075-87.

22. Fitzpatrick ME, Tedrow JR, Hillenbrand ME, Lucht L, Richards T, Norris KA, et al. Pneumocystis jirovecii colonization is associated with enhanced Th1 inflammatory gene expression in lungs of humans with chronic obstructive pulmonary disease. Microbiol Immunol. 2014;58(3):202-11.

23. Mekinian A, Durand-Joly I, Hatron PY, Moranne O, Denis G, DeiCas E, et al. Pneumocystis jirovecii colonization in patients with systemic autoimmune diseases: prevalence, risk factors of colonization and outcome. Rheumatology (Oxford, England). 2011;50(3):569-77.

24. Sheikholeslami MF, Sadraei J, Farnia P, Forozandeh M, Emadi Kochak H, Tabarsi P, et al. Colonization of Pneumocystis jirovecii in Chronic Obstructive Pulmonary Disease (COPD) patients and the rate of Pneumocystis pneumonia in Iranian non-HIV(+) immunocompromised patients. Iran J Microbiol. 2013;5(4):411-7.

25. Respaldiza N, Medrano FJ, Medrano AC, Varela JM, de la Horra C, Montes-Cano M, et al. High seroprevalence of Pneumocystis infection in Spanish children. Clin Microbiol Infect. 2004;10(11):1029-31. 
26. Vargas SL, Ponce CA, Galvez P, Ibarra $\mathrm{C}$, Haas EA, Chadwick AE, et al. Pneumocystis is not a direct cause of sudden infant death syndrome. Pediatr Infect Dis J. 2007;26(1):81-3.

27. Vargas SL, Ponce CA, Luchsinger V, Silva C, Gallo M, Lopez R, et al. Detection of Pneumocystis carinii $\mathrm{f}$. sp. hominis and viruses in presumably immunocompetent infants who died in the hospital or in the community. J Infect Dis. 2005;191(1):122-6.

28. Medrano FJ, Montes-Cano M, Conde M, de la Horra C, Respaldiza N, Gasch A, et al. Pneumocystis jirovecii in general population. Emerg Infect Dis. 2005;11(2):245-50.

29. Ponce CA, Gallo M, Bustamante R, Vargas SL. Pneumocystis colonization is highly prevalent in the autopsied lungs of the general population. Clin Infect Dis. 2010;50(3):347-53.

30. Huang L, Crothers K, Morris A, Groner G, Fox M, Turner JR, et al. Pneumocystis colonization in HIVinfected patients. J Eukariot Microbiol. 2003;50 Suppl:616-7.

31. Gutiérrez S, Morilla R, León JA, Martín-Garrido I, Rivero L, Friaza V, et al. High prevalence of Pneumocystis jiroveci colonization among young HIVinfected patients. J Adolesc Health.. 2011;48(1):103-5.

32. Morris A, Kingsley LA, Groner G, Lebedeva IP, Beard CB, Norris KA. Prevalence and clinical predictors of Pneumocystis colonization among HIV-infected men. AIDS (London, England). 2004;18(5):793-8.

33. Probst M, Ries H, Schmidt-Wieland T, Serr A. Detection of Pneumocystis carinii DNA in patients with chronic lung diseases. Eur J Clin Microbiol Infect Dis. 2000;19(8):644-5.

34. Respaldiza N, Montes-Cano MA, Dapena FJ, de la Horra
C, Mateos I, Medrano FJ, et al. Prevalence of colonisation and genotypic characterisation of Pneumocystis jirovecii among cystic fibrosis patients in Spain. Clin Microbiol Infect. 2005;11(12):1012-5.

35. Gal SL, Hery-Arnaud G, Ramel S, Virmaux M, Damiani C, Totet A, et al. Pneumocystis jirovecii and cystic fibrosis in France. Scand J Infect Dis. 2010;42(3):225-7.

36. Calderón EJ, Regordan C, Medrano FJ, Ollero M, Varela JM. Pneumocystis carinii infection in patients with chronic bronchial disease. Lancet. 1996;347 (9006):977.

37. Vidal S, de la Horra C, Martín J, Montes-Cano MA, Rodríguez E, Respaldiza N, et al. Pneumocystis jirovecii colonisation in patients with interstitial lung disease. Clin Microbiol Infect. 2006;12(3):231-5.

38. Wang DD, Zheng MQ, Zhang N, An CL. Investigation of Pneumocystis jirovecii colonization in patients with chronic pulmonary diseases in the People's Republic of China. Int J Chron Obstruct Pulmon Dis. 2015;10:2079-85.

39. Calderón EJ, Rivero L, Respaldiza $\mathrm{N}$, Morilla R, Montes-Cano MA, Friaza V, et al. Systemic inflammation in patients with chronic obstructive pulmonary disease who are colonized with Pneumocystis jiroveci. Clin Infect Dis. 2007;45(2):e17-9.

40. Calderón E, de la Horra C, Medrano FJ, López-Suárez A, MontesCano MA, Respaldiza N, et al. Pneumocystis jiroveci isolates with dihydropteroate synthase mutations in patients with chronic bronchitis. Eur J Clin Microbiol Infect Dis. 2004;23(7):545-9.

41. Morris A, Wei K, Afshar K, Huang L. Epidemiology and 
clinical significance of pneumocystis colonization. J Infect Dis. 2008;197(1):10-7.

42. Kling HM, Shipley TW, Guyach S, Tarantelli R, Morris A, Norris KA. Trimethoprim-sulfamethoxazole treatment does not reverse obstructive pulmonary changes in pneumocystiscolonized nonhuman primates with SHIV infection. J Acquir Inmune Defic Syndr. 2014;65(4):381-9.

43. Fitzpatrick ME, Tedrow JR, Hillenbrand ME, Lucht L, Richards T, Norris KA, et al. Pneumocystis jirovecii colonization is associated with enhanced Th1 inflammatory gene expression in lungs of humans with chronic obstructive pulmonary disease. Microbiol Immun. 2014;58(3):202-11.

44. Morris A, Alexander T, Radhi S, Lucht L, Sciurba FC, Kolls $\mathrm{JK}$, et al. Airway obstruction is increased in pneumocystis-colonized human immunodeficiency virusinfected outpatients. J Clin Microbiol. 2009;47(11):3773-6.

45. Morris A, Sciurba FC, Lebedeva IP, Githaiga A, Elliott WM, Hogg JC, et al. Association of chronic obstructive pulmonary disease severity and Pneumocystis colonization. Am J Respir Crit Care Med. 2004;170(4):408-13.

46. Cosio MG, Saetta M, Agusti A. Immunologic aspects of chronic obstructive pulmonary disease. $\mathrm{N}$ Engl J Med. 2009;360(23):2445-54.

47. Shipley TW, Kling HM, Morris A, Patil S, Kristoff J, Guyach SE, et al. Persistent pneumocystis colonization leads to the development of chronic obstructive pulmonary disease in a nonhuman primate model of AIDS. J Infect Dis. 2010;202(2):302-12.

48. Sivam S, Sciurba FC, Lucht LA, Zhang Y, Duncan SR, Norris KA, et al. Distribution of Pneumocystis jirovecii in lungs from colonized COPD patients. Diagn Microbiol Infect Dis. 2011;71(1):24-8.

49. Khodavaisy S, Mortaz E, Mohammadi F, Aliyali M, et al. Pneumocystis jirovecii colonization in Chronic Obstructive Pulmonary Disease (COPD). Curr Med Mycol. 2015;1(1):42-8.

50. Norris KA, Morris A. Pneumocystis infection and the pathogenesis of chronic obstructive pulmonary disease. Immun Res. 2011;50(2-3):175-80.

51. Morris A, Netravali M, Kling HM, Shipley T, Ross T, Sciurba FC, et al. Relationship of pneumocystis antibody response to severity of chronic obstructive pulmonary disease. Clin Infect Dis. 2008;47(7):e64-8. 